South African Journal of Geomatics, Vol. 6. No. 2, Geomatics Indaba 2017 Special Edition, August 2017

\title{
An evaluation of the efficiency of laser scanning technology in the quantitative analysis of corrosion.
}

\author{
Grobler H.C.I. ${ }^{1}$ and Combrink G. ${ }^{2}$ \\ ${ }^{1}$ University of Johannesburg, South Africa, hgrobler@uj.ac.za \\ 2 University of Johannesburg, South Africa
}

DOI: $\underline{\text { http://dx.doi.org/10.4314/sajg.v6i2.5 }}$

\begin{abstract}
It is estimated that corrosion causes around R260 Billion of damage to infrastructure and equipment in the South African industry annually. Corrosion evaluation and protection in the marine environment is well established. In the mining industry the field of corrosion evaluation is not as well established as it could be. The evaluation and measurement of corrosion rates and physical deterioration of structures through electrochemical measurement, visual inspection and non-destructive test method measuring techniques are well established. The use of sonar (ultrasonic) and laser scanning has been investigated. Laser scanning technology has improved exponentially over the past years and terrestrial and hand-held units are now smaller, more manageable and affordable to the surveyor. Conventional laser scanners are able to measure to an accuracy of millimeters and generate a point cloud of 1 million points per second in some cases. Latest developments in laser scanning include multi-spectral analysis and Red-Green-Blue (RGB) intensity values for each point. Point clouds of data can now be imported into a CAD package and compared to design specifications. In the case where "as-built" specifications differ for the initial design, laser scanning allows the engineer the opportunity to compare and quantify in minute detail the differences between the final structure and the original design. This paper discusses a test during which a corroded and "fouled" pipe was scanned under laboratory conditions using two types of conventional scanners. A field test was performed on two marine vessels to determine the feasibility of laser scanning to complement standard corrosion measuring methods. The application of laser scanning in this field may provide a valuable tool in the detection, measurement and analysis of corrosion in structures.
\end{abstract}




\section{Corrosion and its effects}

Corrosion is often described as the almost inevitable destruction of a material by way of a chemical reaction with its environment, governed by thermodynamics. Unchecked corrosion leads to the wasting away of resources and infrastructure and if left alone may lead to unexpected damage to infrastructure, injury and loss of life. Four elements are essential for corrosion to occur through an electrochemical reaction: -

- The presence of an anode (where we actually observe the loss of material due to oxidation)

- The presence of a cathode (which is typically the driving element for electrochemical reactions to occur at the anode and it is a reduction reaction)

- An electrical conductor to allow the flow of charge via electron flow, and

- An electrolyte that allows the transfer of charge by way of the flow of ions.

Influencing any single (or more) of these four elements will have an influence on the overall corrosion process. Corrosion can be both a localized or uniform (general). Both of these basic forms are undesirable and have wastage and cost implications. However localized corrosion is more insidious than general corrosion as it results in much loss of metal in a small region and often it is not noticed as easily as general corrosion and it can thus lead to catastrophic failures that occur without much perceived warning (Trethewey \& Chamberlain, 1988).

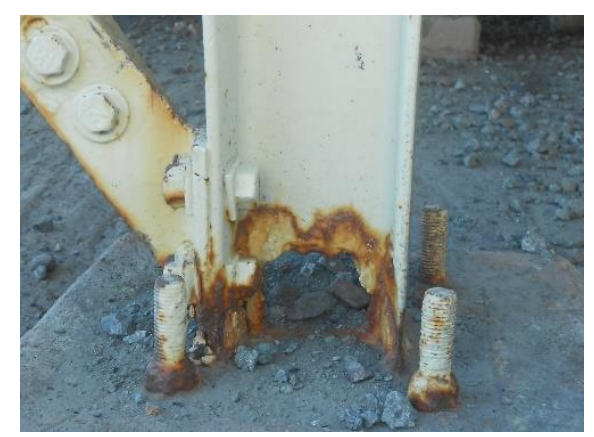

Figure 1. Localized corrosion that is causing the loss of load bearing capacity of a structural beam. How long will it last?

As corrosion is typically inevitable with time, it is essential that infrastructure material be monitored for the degree of damage and possibility of imminent failure that may occur as its lifespan. To this end the structural elements are regularly examined to discover areas that are at risk before the possible envisaged consequential damage becomes a reality. This regular examination of a structure is called "corrosion surveying" and various techniques are employed to generate data from which information is gleaned. 


\section{Traditional corrosion surveying techniques}

The most basic of technique is obviously a visual inspection of the general condition of the structure. This involves noting down where obvious corrosion damage and corrosion products are visible. For some structures, it is often the only technique that is in use today and it can be time consuming if a thorough job is to be done on large structures. It takes experience as sometimes the corrosion products may have been transferred to a location remote from where the corrosion has actually occurred.

Surveying also involves monitoring the condition of anticorrosion technology that has been applied so in the case of cathodic protection of a buried pipeline that is being held at a specific electrical potential, not only is the potential of the pipeline regularly checked but the transformer rectifier and the cables, ground beds, connection points and other associated equipment are also checked. Monitoring the pipe's potential in its basic form and without going into specific details, is typically performed by using a voltmeter and a reference electrode and measuring the voltage between the reference electrode and the pipe at various points along the pipes length. The equipment is itself physically checked and also variation of its settings checked and the influence of such varied settings on the variation in the pipes potential. As the transformer system and its circuits conduct electricity its change in temperature can also provide information on the health of the whole system. An increase in operating temperature of the transformer rectifier unit can be as a result of a higher current demand from the protected pipe or from the circuits of the transformer rectifier units own impending malfunctioning. In the case of the former, an increased current demand can indicate the failure of or damage to the pipes coating system (Combrink, 2017).

In a steel support beam for example corrosion can cause a reduction in the wall thickness and this is often monitored by direct measuring techniques such as using such devices such as rulers (in extreme material losses) Vernier calipers or ultrasonic thickness testers that are used. When the corrosion is in the form of pitting then a profile gauge may instead be used.
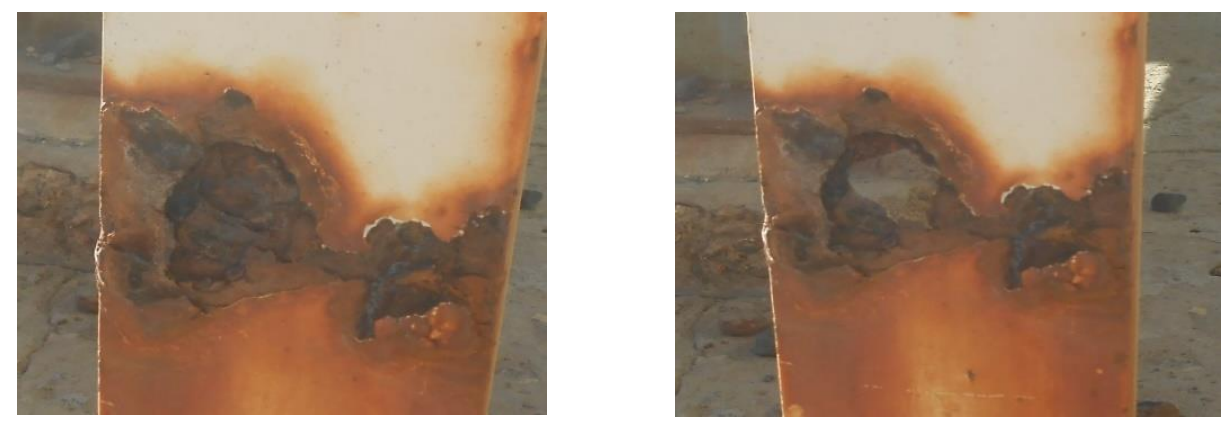

Figure 2 (left). Localized corrosion before the corrosion products have been removed

Figure 3 (right). Localized corrosion after removal of the corrosion products the severity of the damage is evident 
In many cases due to the nature of production or usage corrosion of the structure being surveyed may be obscured and it requires cleaning up before actual the covered metal can be checked.
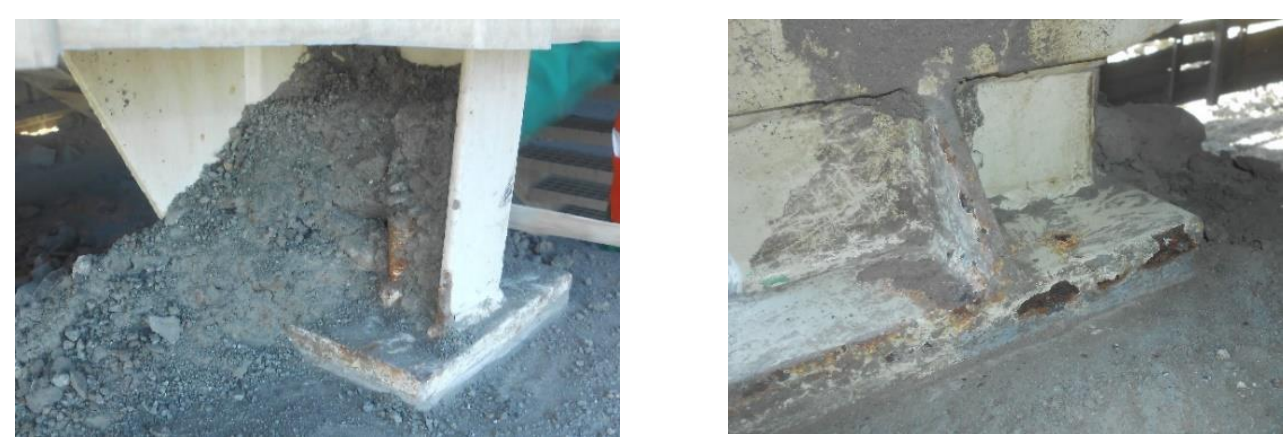

Figure 4 (left). Debris obscuring the metal surface

Figure 5 (right). Corrosion damage evident only after debris has been removed.

Tell-tale visual signs are texture and colour changes the presence of corrosion products on the structure surface and less obvious and typically non-visible degradation signs are loss of adhesion and thinning of the coating. During surveying from a corrosion point of view it is essential to check for such degradation. The visual aspects are relatively easy to detect but to measure progression of degradation over time needs one to perform comparison of earlier conditions with later conditions. This can easily be done if one measured parameters could be digitized and stored for later comparison purpose. In addition, the less obvious degradation can be measured by adhesion testing and film thickness testing and the results can be digitized and also be stored for future comparisons. Special equipment is needed for such measurements (Combrink , 2017).

\section{A brief introduction to laser scanning technology}

Light Detection and Ranging (LIDAR) or laser scanning uses a narrow laser beam to map physical features to a high degree of accuracy. Laser scanners are used to rapidly measure a high density of distances to objects during the measurement the vertical and horizontal angle as well as the beam reflectivity and in some cases multi-spectral information of the object is simultaneously encoded in each observation. The resultant collection of points each of which has a unique $x, y$ and $z$ co-ordinate relative to the laser scanner is referred to as a "point cloud". The strength of the return beam reflected from an object will depend on the surface texture and reflectivity of the object. The reflection co-efficient of the laser beam can be analyzed for multi-spectral characteristics of the object not observed by the naked eye. Phased based scanners, using a constant beam of laser, are able to make more than one million observations per second (3dlasersurveys, 2017), depending on the recommended range, accuracy and computational power of the instrument. Time of flight scanners take less points per second (approximately 50000 points per second) but can work over much longer 
ranges. The accuracy of laser scanners are in most cases within $1 \mathrm{~mm}$, although this is dependent on the type and range of the specific instrument. (Colombo \& Marana, 2010). The accuracy of laser scanning can be influenced by wet surfaces, highly absorbent features such as coal seams. The advantage of an accurate point cloud is that distances can be measured between any of the objects scanned. Laser scanning on its own do not require illumination to measure and can therefore be used in poor lighting conditions. Because the measurement does not require physical contact with the objects surveyed, the scanning of an object will not affect the stability or integrity of an object. A further advantage of laser scanning is that "noise" such as vegetation, dust, moving vehicles or people, can be removed from an image. In a complex multi-level and poorly illuminated environment this capability provides a powerful tool for planning and monitoring purposes. Laser scanners can be mounted on an aircraft (airborne) or on a terrestrial scanner either mounted on a vehicle, backpack or as a conventional survey instrument.

\section{Laboratory experiment.}

An experimental scanning of corroded pipe $540 \mathrm{~mm}$ in length with an internal diameter of $155 \mathrm{~mm}$ and a wall thickness of $5.5 \mathrm{~mm}$ was used within the mining industry was used as a baseline. The pipe was mounted on a rigid platform and scanned using two instruments mounted on a forced-centered beacon. Pipe thickne3ss was measured with an ultrasonic thickness gauge. The pipe thickness could be verified based on the manufacturer's specification and in areas of corrosion material accumulation, thicknesses of up to $19 \mathrm{~mm}$ was measured, from the cross section depicted in Figure 10 these measurements was verified by the laserscan. The purpose of the laser scan was to determine the penetration and accuracy of laser scanning in quantifying the amount of corrosion in a pipe. A high definition Leica P40 scanner (Leica, 2017), with a stated accuracy of $3 \mathrm{~mm}$ up to 50m, was compared with a Leica MS50 multi-station instrument with a stated $0.8 \mathrm{~mm}$ accuracy at $50 \mathrm{~m}$ (Leica, 2007). During testing it was found that the corrosion inside the pipe could be easily defined and measured by the scanner. Some difficulty was experienced with the painted exterior of the pipe. It is considered that the reflectivity of the paint combined with the sensitivity setting of the instruments caused this phenomena. A lower hertz rate of scanning would have increased the coverage. The scan revealed significant detail within the corroded area of the pipe when compare to a visual inspection. Figure 6 is a photograph of the pipe and Figure 7 is a point cloud of the same view of the pipe. 

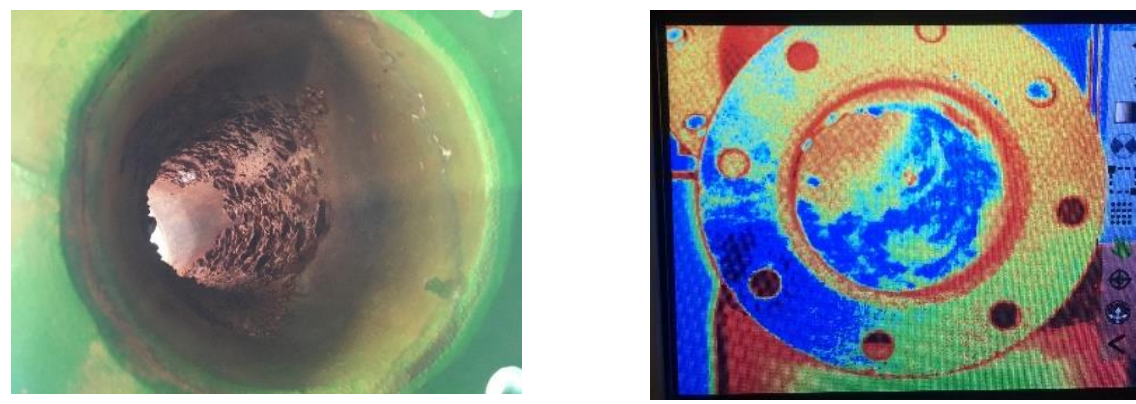

Figure 6 (left). Internal corrosion on a mine pipe

Figure 7 (right). Laser scan of the corrosion of the same pipe

The data was then imported into a basic CAD package which was used to generate sections and 3D views. Using the software measurements of the corrosion and the affected areas can be made. The point cloud image in Figure 8 clearly indicates a wear pattern and areas of accretion of material in the bend of the pipe. These areas can be measured, defined and documented in a CAD package.

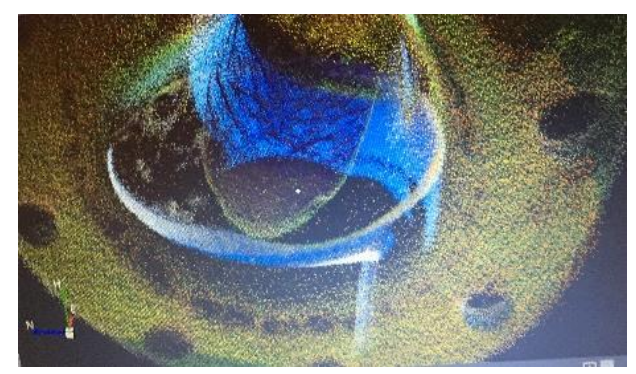

Figure 8. a 3D visual of corrosion in a CAD package, nodes of accretion and wear pattern is observable

A 3D mesh extrusion of external shape and 3D mesh extrusion of internal diameter was created from the point cloud. In order to determine if it would be possible to model corrosion and abrasion patterns, an ideal surface of the pipe based on design parameters was created. Using software technology used in mining to compare tunnel excavations to the design parameters, a comparison between the "ideal" surface and the scanned internal surface was calculated and is indicated in Figure 9. 


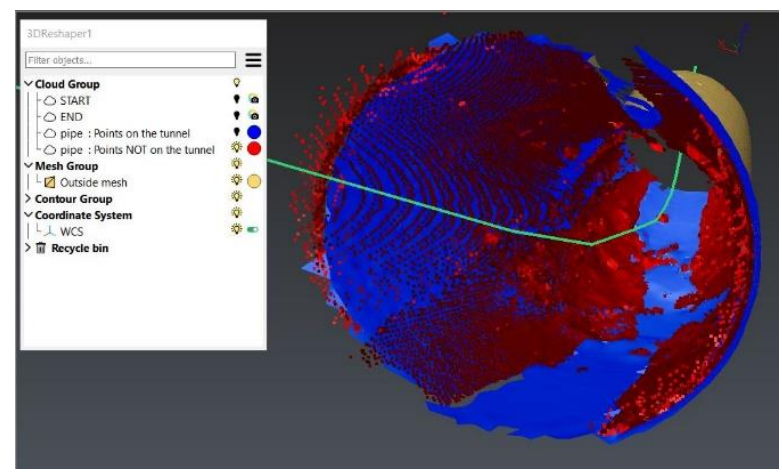

Figure 9. A comparison of the design surface to the actual surface

Using 3D reshaper software (3dreshaper, 2017) cross sections could be drawn and used for comparison. This software provides the option to draw sections through the pipe at any interval, allowing a report of interval sizes to be published for comparison. The original pipe wall thickness is $5 \mathrm{~mm}$, from Figure 10 the amount of accretion of corrosion material can be readily observed.

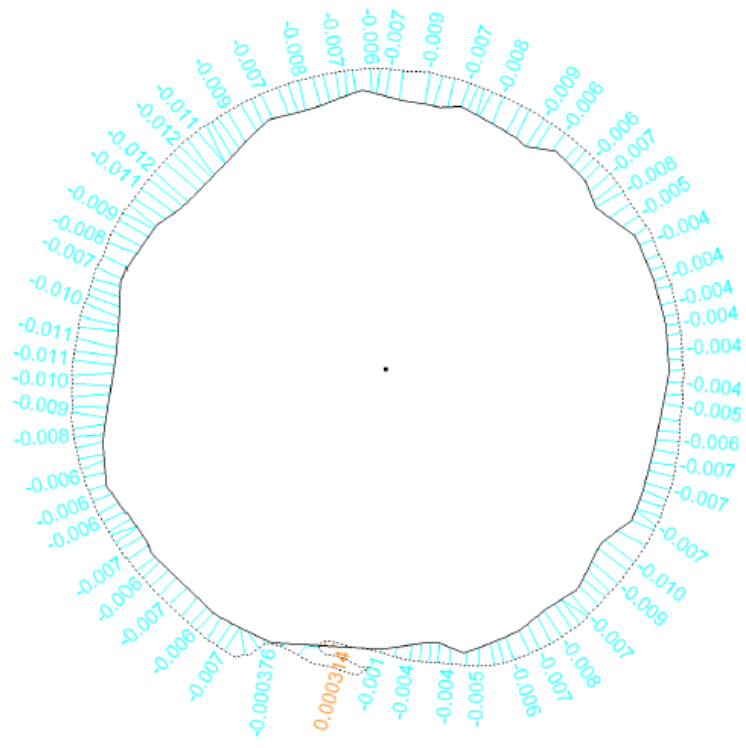

Figure 10 An example for a pipe cross section

These tests indicated that the concept of using laser scanning to quantify and document corrosion and accretion of corrosion products are possible.

\section{Field test}

A test was conducted in a maritime dock yard to assess the feasibility of scanning areas of corrosion on sea-going vessels. A high-definition Z+F 5010C laser scanner (Zoller + Fröhlich GmbH, 2017) was used for the project. The hull of a historical vessel that is part of a museum collection was scanned during the exercise. The vessel with a length of $58 \mathrm{~m}$, standing on a dock was completely scanned in approximately 90 minutes. The detail of the scans made it possible to observe damage to the hull plates of the vessels not clearly visible to the naked eye. 

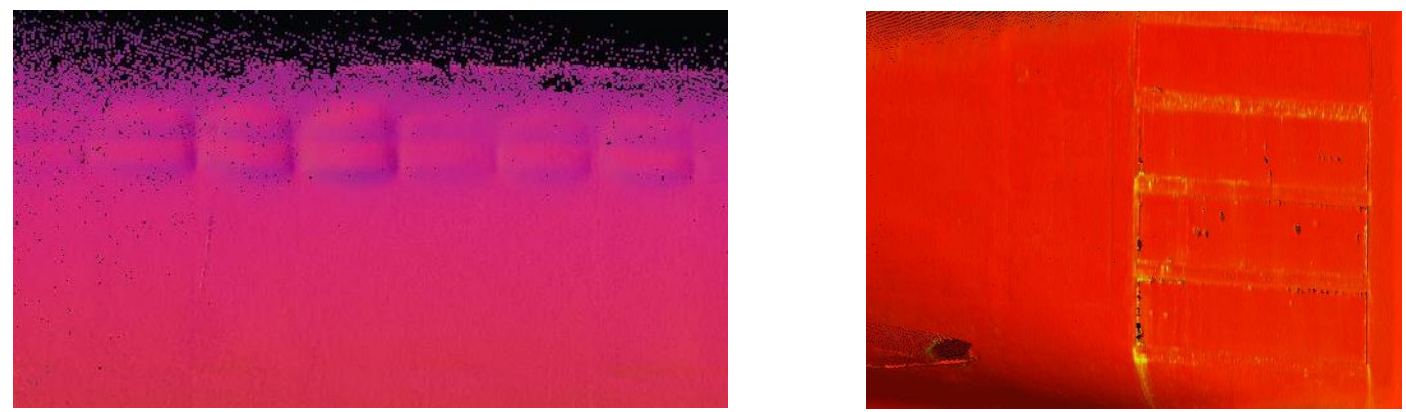

Figure 11 (left). Damage to hull plate on RGB value plot

Figure 12 (right). RGB plot of bow plates not visible to naked eye.

Hull plates covered in paint sometimes obscures detail to the naked eye. In the image Figure 11 and Figure 12, the hull plates can be observed in the "false colour" or "RGB value" (reflectivity strength) image. The areas investigated for corrosion could be easily identified on the scan and quantified. The vessel has an external and internal hull separated by a space that in this case was covered in marine growth and corrosion. The RGB values in

Figure 13 indicates a lower reflectivity of the marine fouling on the internal hull scanned through an access hatch in the external hull. In the cad software a cross section of the same view inFigure 14reveals more detail of the internal and external hull that can be measured and quantified remotely.
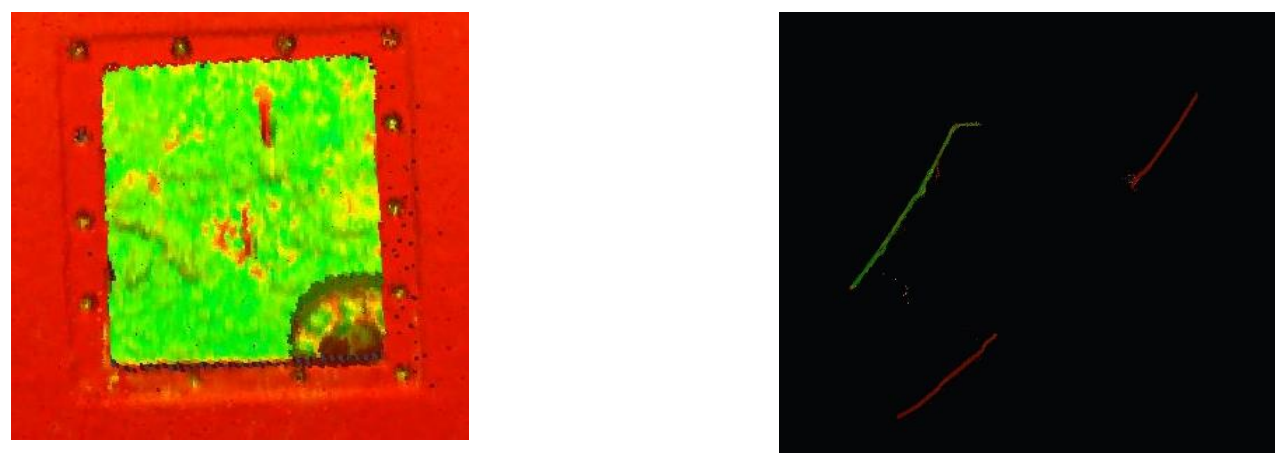

Figure 13 (left). An external scan includes the internal hull structure

Figure 14 (right). A cross section through the external and internal hull-plate

Corrosion patterns observed visually in the dry-dock indicates run-off patterns of corrosions in the image captured during the scanning was measured with the laser scanner and can be quantified in a CAD package. 
South African Journal of Geomatics, Vol. 6. No. 2, Geomatics Indaba 2017 Special Edition, August 2017
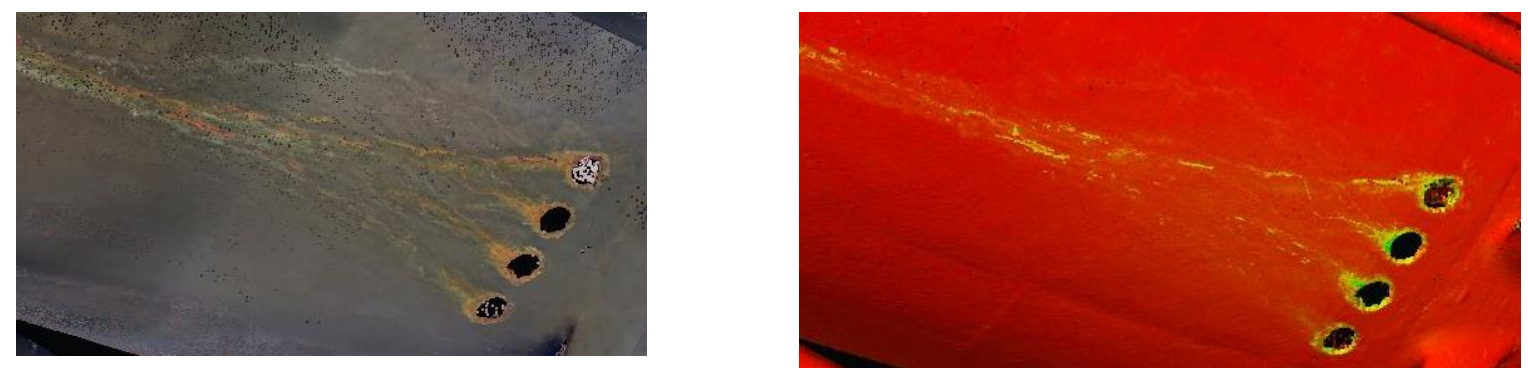

Figure 15 (left). True colour point cloud of the drainage pattern of corrosion on the keel of a vessel Figure 16 (right). Keel corrosion pattern in RGB values

\section{Conclusions made based on the field test}

Corrosion can be quantified through the changes that occur in the medium such as colour-, textureand dimensional changes. These changes are noticeable and measurable and thus laser scanning a surface to map such changes can be beneficial. Laser scans at regular will allow the creation of a three-dimension digital model that can document dimensional, colour and textural changes over time. It is argued that areas of specific interest for corrosion can be measured by a metrology-grade laser scanners In the case of dimension changes some industrial laser scanners can detect changes of 10 microns and this is sufficient for checking corrosion over time of metallic structures. It is suggested that a hybrid of visual inspection, recording by laser scanning and detailed study by conventional corrosion surveying techniques and high accuracy laser scanning in identified areas will allow a comprehensive, measurable record of corrosion.

In addition to traditional 3D scanning, advances in multi-spectral, thermal and full-colour scanning can be performed and a three dimensional hi definition model can be created that shows up differences in dimensions, temperatures, colours and textures and all of these can be correlated with corrosion detected visually or with degradation of protective technology measured in other ways to create a three dimensional picture of degradation due to corrosion and failures of protective systems that will enhance the corrosion surveying of a structure.

The test on a historic vessel provided proof of concept that laser scanning can be used to define corrosion areas on marine vessels. In addition it was found that the laser scan images can reveal hull damage and corrosion not always visible to the naked eye. In the case of repeated scans over a period of time it would be possible to determine corrosion patterns, areas of corrosion that re-occur and document damage to the hull. In addition internal scans of a vessel would be able to determine corrosion and wear patterns on essential equipment. An internal scan of the vessel would assist in documenting all equipment and services such as piping and conduits. Laser scanning provides a 3D point cloud which can be manipulated in conventional draughting packages, as-built surveys and essential repairs on a vessel can be accurately and speedily executed. 


\section{Further advantages of laser scanning on a vessel.}

In addition to the advantages listed above, laser scanning will be able to provide accurate cross sections of a vessel. In cases where a vessel is purchased from a supplier and later outfitted by the enduser, "as-built" surveys of the vessel in its current configuration and cross sections of the vessel would be possible. In the case of damage to the vessel, an accurate idea of the scale of the damage van easily be obtained through a damage assessment scan which can assist in the repair and re-commissioning of the vessel. Hull damage can be determined by sequential scans every time the vessel is in dry dock. All areas above the water line can be scanned for damage and repair purposes while the vessel is underway. Laser scan data can be utilized in a "ship Information Management system" where each part of the vessel is located in 3D space and linked to a database of parts, similar to a Building Information Management system.

\section{References}

3dlasersurveys. (2017, June 1). Frequently asked Questions. Retrieved from 3dlasersurveys: http://3dlasersurveys.com/about-us/faqs

3dreshaper. (2017). 3dreshaper. Retrieved from plug in: http://www.3dreshaper.com/en/

Colombo, L., \& Marana, B. (2010, December 3). Terrestrial Laser Scanning . Retrieved from GIM International: http://www.gim-international.com/content/article/terrestrial-laser-scanning-2

Combrink , G. A. (2017). A Short Commentary on The Corrosive Nature of The Mining Environment at "mine $x$ " and its effect on the internal corrosion of the roofbolts. Johannesburg: Combrink.

Leica. (2007).Leica_Nova_MS50_DAT_us.pdf. Retrieved from leicageosystemsdares: http://www.leicageosystemsdares.com/download/Leica_Nova_MS50_DAT_us.pdf

Leica. (2017, July 1). leica-scanstation-p40. Retrieved from leica-geosystems: http://leicageosystems.com/products/laser-scanners/scanners/leica-scanstation-p40--p30

Smolders, M., \& GÖrtz, H. (2016, March ). Indoor wayfinding at Amsterdam airport. GIM International, pp. 16-18.

Trethewey, K. R., \& Chamberlain, J. (1988). Corrosion for students and engineering. Singapore: Longman scientific and technical.

wikipedia. (2016, May). Simultaneous_localization_and_mapping. . Retrieved from wikipedia.org: https://en.wikipedia.org/wiki/Simultaneous_localization_and_mapping. 
South African Journal of Geomatics, Vol. 6. No. 2, Geomatics Indaba 2017 Special Edition, August 2017

Woods, S. (2016, March). Laser scanning on the go. GIM International, pp. 29-31.

Zoller + Fröhlich GmbH. (2017). Z-F-IMAGER-R-5010C.3d_laserscanner. Retrieved from zf-laser: http://www.zf-laser.com/Z-F-IMAGER-R-5010C.3d_laserscanner.0.html?\&L=1 